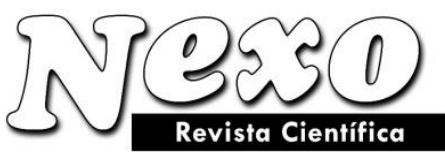

Vol. 34, No. 02, pp. 993-1007/Junio 2021

\title{
Monitoring optimization of telecommunication networks for generation of reserve capacity in system
}

\section{Optimización del seguimiento de las redes de telecomunicaciones para la generación de capacidad de reserva en el sistema}

\author{
Oleg Jakovlevich Kravets ${ }^{1, *}$, Elena Ivanovna Danilina ${ }^{2}$, Iana Ivanovna Malikova ${ }^{3}$, Dmitry \\ Vladimirovich Gorelov ${ }^{4}$ \\ ${ }^{1}$ Voronezh State Technical University, Voronezh, Russia. \\ ${ }^{2}$ Russian University of Transport (MIIT), Moscow, Russia. \\ ${ }^{3}$ Vologda state University, Vologda, Russia. \\ ${ }^{4}$ Institute of Business Career, Moscow, Russia. \\ *kravets.o.ja@mail.ru
}

(recibido/received: 17-febrero-2021; aceptado/accepted: 18-abril-2021)

\begin{abstract}
This article discusses an approach to monitoring optimization in telecommunication networks with packet routing. Mathematical model of data networking with accounting for monitoring traffic structure is described, optimization task is determined. Main attention is paid to model development on the basis of queueing theory in order to generation of reserve capacity in system. The main concept is that comparatively simple structure of monitoring network is imposed on physical structure of telecommunication network. The modeling is aimed at selection of optimum parameters of monitoring system, solution techniques for similar task are proposed. The obtained optimization task is sufficiently complicated, subsequent attempts should be aimed at searching for conditions which would enable introduction of some constraints in order to simplify its solution without noticeable loss of the model adequacy. This is especially important for asynchronous task of monitoring, it should be based on selection of the most acceptable type of objective function (linear or quadratic). Management system structure of terminal equipment monitoring has been developed for railway sector on the basis of SNMP V3. The system would permit to improve confidence level of data received from measuring system about electrical parameters of route cables, thus decreasing human errors upon classification of emergency situations occurring in communication networks.
\end{abstract}

Keywords: Network Monitoring, Mathematical Model, Monitoring Optimization, Distributed System.

\section{RESUMEN}

Este artículo analiza un enfoque para monitorear la optimización en redes de telecomunicaciones con enrutamiento de paquetes. Se describe el modelo matemático de redes de datos con contabilidad para monitorear la estructura del tráfico, se determina la tarea de optimización. Se presta especial atención al desarrollo de modelos sobre la base de la teoría de las colas para generar capacidad de reserva en el 
sistema. El concepto principal es que la estructura comparativamente simple de la red de monitoreo se impone a la estructura física de la red de telecomunicaciones. El modelado tiene como objetivo la selección de los parámetros óptimos del sistema de monitoreo, se proponen técnicas de solución para tareas similares. La tarea de optimización obtenida es suficientemente complicada, los intentos posteriores deben estar dirigidos a buscar condiciones que permitan la introducción de algunas restricciones para simplificar su solución sin una pérdida notable de la adecuación del modelo. Esto es especialmente importante para la tarea asincrónica de monitoreo, debe basarse en la selección del tipo de función objetivo más aceptable (lineal o cuadrática). La estructura del sistema de gestión de la monitorización de equipos terminales se ha desarrollado para el sector ferroviario sobre la base de SNMP V3. El sistema permitiría mejorar el nivel de confianza de los datos recibidos del sistema de medición sobre los parámetros eléctricos de los cables de ruta, disminuyendo así los errores humanos al clasificar las situaciones de emergencia que ocurren en las redes de comunicación.

Palabras clave: Monitoreo de Red, Modelo Matemático, Optimización de Monitoreo, Sistema Distribuido.

\section{INTRODUCTION}

While building data communication systems (DCS), at the stage of development as well as at the stage of implementation it is necessary to account for management of DCS devices of telecommunication network. In large networks such task is non-trivial and should be analyzed at designing stage. This task should be based on development and solution of mathematical model of designed network with accounting for problems of packet routing and switching, as well as accounting for packets and disturbances induced by monitoring systems, solution of monitoring optimization task and analysis of monitoring frequency for various situations.

The main concept is that comparatively simple structure of monitoring network is imposed on physical structure of telecommunication network. Monitoring system can be a subset of telecommunication network, or can be implemented on the basis of techniques differing from basic network. As a consequence, Markov network models can be accepted, then it becomes possible to obtain exact analytical description of optimization task.

In Section 2, we review the previous work in DSC monitoring. Section 3 addresses the question of useful DSC modeling features. The task is formalized in terms of queueing systems. A model of data transfer system is built, where each channel is presented by two server facilities, and network nodes are preset by switching matrices for flow parameter association. Given the discovered mathematical models, Section 4 presents the optimization problem and discussion about its solving. System architecture on the basis of Simple Network Management Protocol (SNMP) (aimed at solution of monitoring task for terminal equipment in railway sector) is described in Section 5.

\section{RELATED WORK}

Traditional heterogeneity of both telecommunication systems, computer networks, network data resources, and the range of their users complicates non-prejudicial monitoring and analysis of telecommunication architectures and resources. In this regard, while operating telecommunication systems and computer networks, it is required to apply sufficiently wide range of modern engineering solutions of their monitoring and analysis (for example, radio monitoring (Spajic, Kozic, Pokrajac and Okiljevic, 2012), simulation of telecommunication systems (Lencse, Derka and Muka, 2013), multi-levels heterogeneous routing (S. Tyagi, S. Tanwar, S.K. Gupta et al, 2015), automatic monitoring \& detection system for grey traffic (Khan, Imtiaz and Shakir, 2015), monitoring services for scalable heterogeneous distributed 
systems (Subramanian et al.,, 2006), which would improve efficiency of operation of telecommunication networks.

Analysis of such systems is usually based on Queueing System theory (Romansky, 2013), various approaches are applied - both direct analytical description with a focus on particular targeted region (Norris, 1997), and more common application for Markov flow modeling of High-Reliability Telecommunications Systems ( Kitchin, 2006) - for network flows analysis (Morley and Thornes, 2010), in large-scale (military) personnel systems (Merck and Hall, 1971), for transport of mixed-size sediment particles under unsteady flow conditions ¿Error! No se encuentra el origen de la referencia.Kuai and Tsai, 2016), for analysis of waiting time process of Markov type (Clarke, 1957). From the point of view of theoretical description, communication channels are interpreted as edges of graph, which describes the system, and are passive elements. In this article we propose another approach. Moreover, we can't find works with generation of reserve capacity in system.

The tasks, obtained as a consequence of analytical description, are solved both by conventional optimization methods (Bandi, 1988, Fletcher, 2000), and by heuristics. The range of methods is rather wide and makes it possible to achieve practical solution of nearly all optimization scenarios.

Total variety of tools used for application analysis of diagnostics of computational networks can be subdivided into several large classes.

1. Management system agents supporting functions of one of conventional MIB (Management Information Bases) and transferring data over SNMP or CMIP (Common Management Information Protocol) (Pandey, Choi, Won and Won-Ki Hong, 2011). In order to receive data from agents, manage system is usually required, which automatically collects data from agents.

2. Embedded systems. These systems are made in the form of firmware modules embedded into switching equipment, as well as in the form of software modules embedded into operation systems (Subramanian et al, 2006). They perform functions of diagnostics and management of a single device, this is their main difference from centralized management systems. Facilities of this class can be exemplified by management module of multisegment repeater Ethernet, which performs functions of autosegmentation of ports upon detection of faults, assigning of ports to internal segments of repeater, and some other functions. Generally, the embedded management modules also act as SNMP agents transferring data on device state for management systems (Ahn and Chung, 2005).

3. Protocol analyzers. They are soft- or firmware systems, which, contrary to management systems, are limited only to monitoring functions and traffic analysis in networks. Good protocol analyzer can capture and decode packets of numerous protocols applied in networks - about several tens. Protocol analyzers make it possible to set certain logic conditions of capturing of individual packets and perform complete decoding of captured packets, that is, show in user convenient form nesting packets of different level protocols with deciphering of contents of individual fields of each packet.

The concept of this article is that comparatively simple structure of monitoring network is imposed on physical structure of telecommunication network. Monitoring system can be a subset of telecommunication network, or can be implemented on the basis of techniques differing from basic network. As a consequence, Markov network models can be accepted, it becomes possible to obtain exact accurate analytical description of optimization task.

\section{MATHEMATICAL SIMULATION OF DATA COMMUNICATION SYSTEM IN MONITORING SUBSET}




\subsection{Formalization of the task}

Data flow diagram of the considered system is illustrated in Fig. $1\left(\lambda^{(0)}\right.$-common flow, $\lambda^{(1)}$-monitoring station flow, $\lambda^{(2)}$-flow from controlled devices, Mon. $=$ monitoring station).

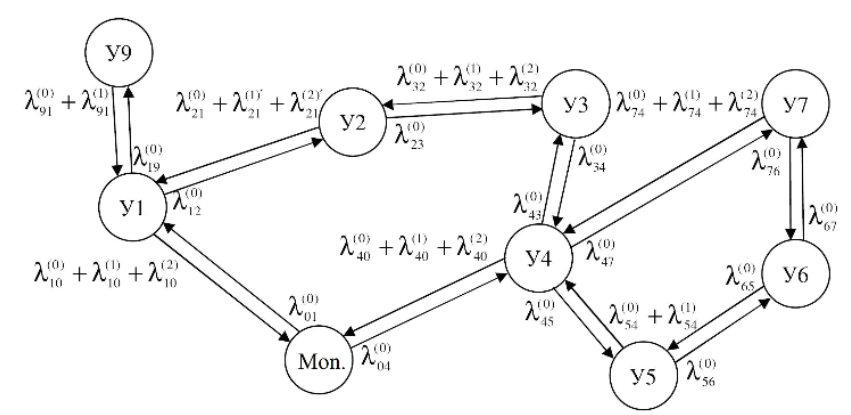

Figure 1. Data flow diagram in data communication system.

Let us consider data networking from the point of view of its description in terms of queueing systems (QS). In this model messages sequentially pass via some nodes (switching devices). Such approach makes it possible to consider data communication system as Network of queuing systems (Jackson, 1957), where each QS can be considered separately, but all of them are characterized by interrelations between their parameters. For illustration purposes below is given the simplest matrix of intensities of internode flows corresponding to Fig. 2, where only flows between nodes $\mathrm{i}-1, \mathrm{i}, \mathrm{i}+1$ and $\mathrm{i}+2$ are depicted. Intersection between column $\mathbf{k}$ and line $\mathbf{I}$ shows flow intensity corresponding to flow from node $\mathbf{k}$ to node $\mathbf{l}$. In Fig. 2 the flows between nodes $\mathbf{i}$ and $\mathbf{i}+\mathbf{1}$ are depicted with accounting for elements of switching matrix, which characterize intensity distribution of input flows in output flows for given node:

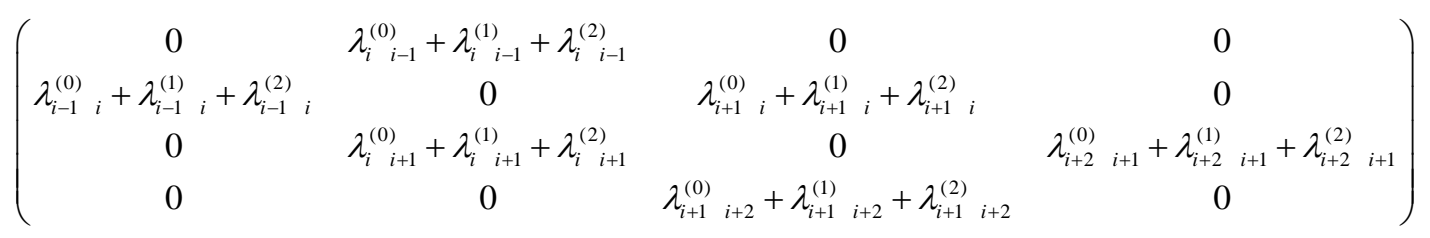

While using QS theory as a model, it is required to determine for each included device what is a queue block, a service device, and what is request source. In such cases I/O exchange buffers serve as queue blocks.

Up-to-date DCS devices are equipped with sufficiently large buffers, and in combination with modern data transfer protocols this provides possibility to consider all DCS devices as having unlimited I/O buffers. Similar assumption makes it possible to use QS with unlimited queue length (the model considered below does not imply any constraints on QS type, and application of this constraint can be useful for task solution (Kingston, 1983)).

Service devices are data transfer channels, all of them are characterized by certain throughput. Each channel can be presented by one or two independent service devices. 


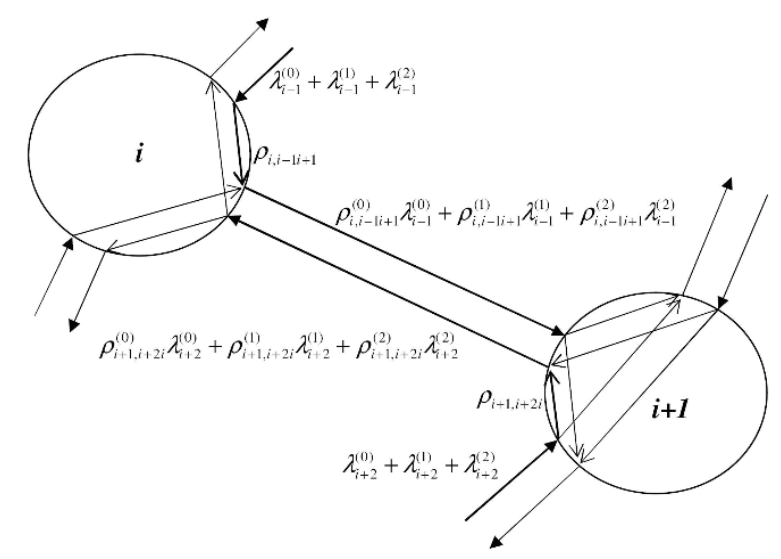

Figure 2. Interrleation between QS parameters

Request sources in DCS model are concentrators of local networks or single devices (servers, network printers, and so on) connected directly to ports of commutating switches and routers.

\subsection{Model of data networking system}

Now, let us consider a model where each channel is presented by two service devices, and network nodes preset switching matrices for interrelation between flow parameters (Fig. 1).

The values of switching matrix are taken from known intensities of request flows (packets) and their distribution at each commutating switch (router). Such description sufficiently well characterizes the process of switching and static routing. In practice, large networks usually operate with more complex protocols such as RIP (Routing Information Protocol), OSPF (Open Shortest Path First), EIGRP (Enhanced Interior Gateway Routing Protocol) and others, however, if the process of rerouting is not considered, and it should be infrequent, then such model can also serve as adequate description for such DCS.

More exact description is required by necessity to separate flows generated by request flows. In order to solve this task, it was proposed to detach background flow of requests and flows related with monitoring of devices (Fig. 3). Such separation is required for assigning of individual routes and traffic analysis generated by requests from monitoring station and responses from devices.

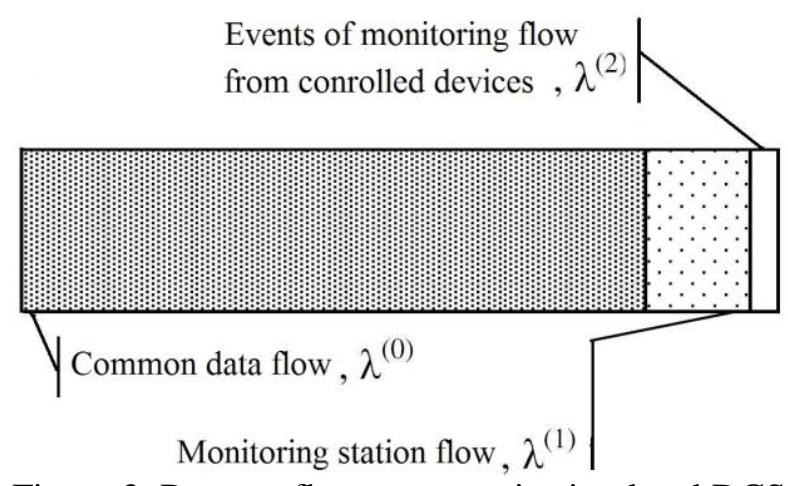

Figure 3. Request flow structure in simulated DCS

Input parameters for node are flow intensities $\lambda_{\mathrm{i}, \mathrm{j}}$, where $\mathbf{i}$ is the outcoming flow node index, $\mathbf{j}$ is the incoming node index. Different nodes have different number of inputs/outputs, let us denote it as $\mathrm{m}_{\mathrm{i}}$, 
where $\mathbf{i}$ is the node index. Another node characteristics are elements of switching matrices $-\rho_{\mathrm{i}, \mathrm{k}}$, where $\mathbf{i}$ is the node index, and $\mathbf{k , ~} \mathbf{l}$ are input/output via which the flow passes (Fig. 4). Then, the intensity of flow from the $\mathrm{i}$-th node to the $\mathrm{j}$-th node can be presented as follows:

$$
\lambda_{i j}=\sum_{k=1}^{m_{i}} \rho_{i, f(k, i) j} \lambda_{f(k, i), i}
$$

where $f\left(i_{1}, i_{2}\right)$ is the function which preset allocation of input and output indices, it was introduced just to simplify the order of their numeration. Therefore, summation over all I/O was performed.

Going to node in total, this equation can be presented in matrix form using the following switching matrix:

$$
P_{i}=\left(\begin{array}{ccc}
\rho_{i, f(1, i) f(1, i)} & \cdots & \rho_{i, f(1, i) f\left(m_{i}, i\right)} \\
\vdots & \ddots & \vdots \\
\rho_{i, f\left(m_{i}, i\right) f(1, i)} & \cdots & \rho_{i, f\left(m_{i}, i\right) f\left(m_{i}, i\right)}
\end{array}\right),
$$

and flow intensity vector for the i-th node:

$$
\Lambda_{i}=\left(\begin{array}{c}
\lambda_{f(1, i), i} \\
\vdots \\
\lambda_{f\left(m_{i}, i\right), i}
\end{array}\right)
$$

Then, Eq. (2) with accounting for overall node can be presented as follows:

$$
\lambda_{i f(k, i)}=P_{i}^{f(k, i)} \Lambda_{i}
$$

Total system for all $\mathbf{n}$ nodes with $\mathbf{m}_{\mathbf{i}}$ inputs/outputs will be described by the following set of linear algebraic equations:

or

$$
\lambda_{i f(k, i)}=P_{i}^{f(k, i)} \Lambda_{i}, i=\overline{1, n} ; k=\overline{0, m_{i}}
$$

$$
\begin{gathered}
\lambda_{i f(k, i)}=\rho_{i, f(1, i) f(k, i)} \lambda_{f(1, i) i}+\ldots+\rho_{i, f\left(m_{i}, i\right) f(k, i)} \lambda_{f\left(m_{i}, i\right) i}, \\
i=\overline{1, n}, k=\overline{0, m_{i}} .
\end{gathered}
$$




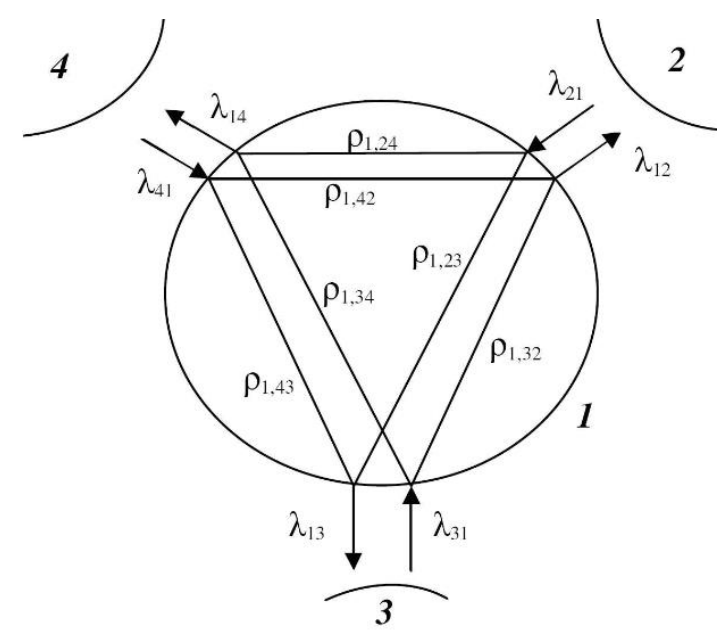

Figure 4. Schematic diagram of DCS unit and interrelations between flows.

The simulation is aimed at study of the system upon various behavior of DCS monitoring systems, which introduce additional data flow into common network traffic. Since this flow is not related with common data flows, it would be reasonable to introduce separate intensities for this flow, i.e. one more set of equations is required, which will describe distribution of monitoring system traffic. In its turn, the monitoring task is subdivided into two constituents: active monitoring by certain controlling station and data sent by DCS devices. Then, complete intensity of all flows is:

$$
\lambda=\lambda^{(0)}+\lambda^{(1)}+\lambda^{(2)}
$$

where $\lambda^{(0)}$ is the intensity of common flow, $\lambda^{(1)}$ is the intensity of monitoring station flow, $\lambda^{(2)}$ is the intensity of event flow from devices:

$$
\begin{gathered}
\lambda_{i f(k, i)}^{(0)}=P_{i}^{f(k, i)^{(0)}} \Lambda_{i}^{(0)}, \\
\lambda_{i f(k, i)}^{(1)}=P_{i}^{f(k, i)^{(1)}} \Lambda_{i}^{(1)}, \\
\lambda_{i f(k, i)}^{(2)}=P_{i}^{f(k, i)^{(2)}} \Lambda_{i}^{(2)}, \\
i=\overline{1, n} ; k=\overline{0, m_{i}} .
\end{gathered}
$$

It is required to consider the task with non-stationary flows. In the considerations below direct mentioning of flow parameters as function of time can be omitted but it will be implied.

A continuous-time Markov chain will be considered as model (Norris, 1997), which describes birth and death with infinite number of states (S) (Fig. 5). Let use "direct" equation of birth and death:

$$
\begin{gathered}
P_{i n}^{\prime}(t)=\frac{d P_{i n}(t)}{d t}=-\left(\lambda_{n}+\mu_{n}\right) P_{i n}(t)+{ }_{+} \lambda_{n-1} P_{i, n-1}(t)+\mu_{n+1} P_{i, n+1}(t), \\
t \geq 0, n>0, i=0,1,2, \ldots ; \\
P_{i 0}^{\prime}(t)=\frac{d P_{i 0}(t)}{d t}=-\lambda_{0} P_{i 0}(t)-\mu_{1} P_{i 1}(t), \\
n=0, i=0,1,2, \ldots
\end{gathered}
$$


Hereafter the index $\mathbf{i}$, which characterizes initial state, is omitted but it will be implied.

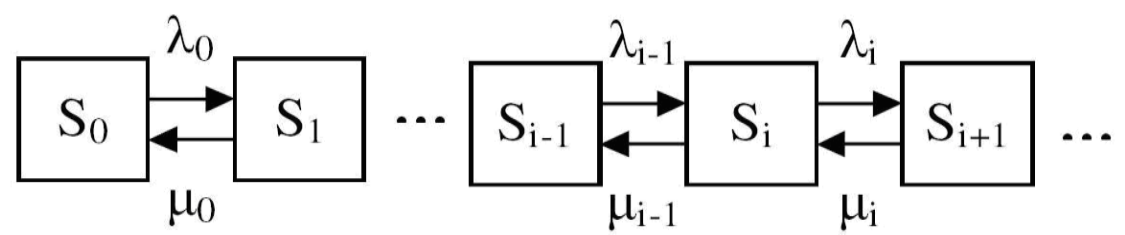

Figure 5. State graph of death and birth.

While analyzing and solving this task, the parameters of which depend on time, it would be reasonable to consider their dependence as periodic (similar task was solved in (Norris, 1997)). This statement does not limit generality. Firstly, in actual networks traffic has periodic structure (the most distinct periodicity - by days). Secondly, if non-periodic dependence is of interest, then it is always possible to expand our function to periodic with period equaling to our interval of concern, and the obtained solution can be studied in this interval. Let us introduce the following transformation of time $\tau$ :

$$
\tau \equiv \tau(t)=\int_{0}^{t} \mu(s) d s
$$

In order to simplify calculations, let us apply the time scale $\tau$ :

$$
\begin{gathered}
\rho(\tau)=\frac{\lambda(t)}{\mu(t)}, \\
R(\tau)=\frac{\int_{0}^{t} \lambda(s) d s}{\int_{0}^{t} \mu(s) d s}=\frac{1}{\tau} \int_{0}^{\tau} \rho(s) d s .
\end{gathered}
$$

Substituting Eq. (19) into Eq. (20), we obtain "direct" equation of death and birth with new scaling variable $\tau$ :

$$
\begin{aligned}
& \frac{P_{0}(\tau)}{d \tau}=-\rho(\tau) P_{0}(\tau)+P_{1}(\tau) \\
& \frac{P_{n}(\tau)}{d \tau}=-[1+\rho(\tau)] P_{n}(\tau)+\rho(\tau)+\rho(\tau) P_{n-1}(\tau)+P_{n+1}(\tau), n>0 . \\
& \text { Let } Q_{n}(\tau)=e^{\tau[1+R(\tau)]} P_{n}(\tau), \mathrm{n}=0,1, \ldots, \text { then the set (22) will be as follows: } \\
& \frac{d Q_{0}(\tau)}{d \tau}=Q_{0}(\tau)+Q_{1}(\tau) \\
& \frac{d Q_{n}(\tau)}{d \tau}=\rho(\tau) Q_{n-1}(\tau)+Q_{n+1}(\tau), n>0 \text {. }
\end{aligned}
$$

In order to solve this set, it is necessary to convert it to partial differential equation using the method of generating functions. Applying the generating function: 


$$
Q(z, \tau)=\sum_{n=0}^{\infty} Q_{n}(\tau) \frac{(z-\tau)^{n}}{n !}
$$

we obtain the following equation:

$$
\frac{\partial^{2} Q}{\partial \tau \partial z}=\rho(\tau) Q
$$

Differentiating $Q(z, \tau)$ with respect to $\tau$, setting $\mathrm{z}=\tau$ and applying equation for $Q_{0}^{\prime}(\tau)$ from Eq. (24), we obtain the boundary conditions:

$$
\left.\frac{\partial Q(z, \tau)}{\partial \tau}\right|_{z=\tau}=Q(\tau, \tau)
$$

From the condition of initial system state we determine that $Q(z, 0)=\frac{z^{i}}{i !}$, and let $f(\tau)=\frac{\partial Q(0, \tau)}{\partial \tau}$.

Solution of Cauchy problem for hyperbolic equation we obtain using Riemann functions:

$$
Q(z, \tau)=A_{i}(0, \tau, z)+\int_{0}^{\tau} A_{0}(\sigma, \tau, z) f_{i}(\sigma) d \sigma
$$

where:

$$
\begin{gathered}
A_{n}(\sigma, \tau, z)=z^{\frac{n}{2}}\left[R_{\sigma, \tau}\right]^{-\frac{n}{2}} I_{0}\left(2\left\{R_{\sigma, \tau} z\right\}^{\frac{1}{2}}\right), n=0, \pm 1, \ldots ; \\
\frac{\partial A_{n}(\sigma, \tau, z)}{\partial z}=A_{n-1}(\sigma, \tau, z) \\
\frac{\partial A_{n}(\sigma, \tau, z)}{\partial \tau}=\rho(\tau) A_{n+1}(\sigma, \tau, z) \\
A_{n}(0,0,0,)=\delta_{0 n}, \\
A_{-n}(\tau, \tau, \tau)=\left\{\begin{array}{l}
0, n>0, \\
1, n=0 .
\end{array}\right. \\
B_{n}(\sigma, \tau)=A_{n}(\sigma, \tau, \tau)-\rho(\tau) A_{n+1}(\sigma, \tau, \tau), \\
f_{i}(\tau)=B_{i}(0, \tau)+\int_{0}^{\tau} B_{0}(\sigma, \tau) f_{i}(\sigma) d \sigma \\
R_{\sigma, \tau}=R(\tau) \tau-R(\sigma) \sigma .
\end{gathered}
$$

Using these expressions, we proceed from generating function $\mathrm{Q}(\mathrm{z}, \tau)$ to the required one:

$$
P_{n}(\tau)=\exp \{-\tau[1+R(\tau)]\}\left[A_{i-n}(0, \tau, \tau)+\int_{0}^{\tau} \mathrm{A}_{-\mathrm{n}}(\sigma, \tau, \tau) f_{i}(\sigma) d \sigma\right]
$$


Knowing conventional probabilities that at the instant of time $\tau$ there are $\mathbf{n}$ packets in channel (provided that at $\tau=0$ there were $\mathbf{i}$ packets) (11) and distribution density of waiting time of the $(n+1)$-th packet, average waiting time of a packet in queue $\mathrm{Lq}_{\mathrm{ij}}$ for this channel and dispersion of waiting time $\mathrm{Dq}_{\mathrm{ij}}$ can readily be obtained. Therefore, determining all conventional probabilities (37), we obtain the set of variables $\mathrm{Lq}_{\mathrm{ij}}$ and $\mathrm{Dq}_{\mathrm{ij}}$, which characterize loading of nodes (routers). Setting certain range of permissible variation of these variables in monitoring and solving optimization task, we can determine optimum monitoring frequency.

\section{MONITORING OPTIMIZATION}

Selection of criterion of data networking monitoring is reduced to maximization of monitoring frequency $f_{\text {mon }}$ (for one controlling station). Hereafter we will use the notation $f \equiv f_{\text {mon }}$. Herewith, the following conditions should be met: condition of "minimum disturbance" (monitoring system flow increases average waiting time more than by $\zeta$ ) and condition of "uniformity" (dispersion of average waiting time should increase not more than by $\eta$ ).

$$
\begin{gathered}
\operatorname{Lq}_{i j}^{m o n}(f) \leq(1+\varsigma) L q_{i j}, \\
D q_{i j}^{m o n}(f) \leq(1+\eta) D q_{i j} \\
\quad i=\overline{1, n}, j=\overline{1, n} .
\end{gathered}
$$

It should be mentioned that the average service time $\mathrm{Lq}$ is determined without monitoring flow, that is, we consider only $\lambda^{(0)}$, whereas $\mathrm{Lq}^{\text {mon }}$ with consideration for total flow is $\lambda=\lambda^{(0)}+\lambda^{(1)}+\lambda^{(2)}$; the same for dispersions Dq, Dq ${ }^{\mathrm{mon}}$.

It can be seen that this is the simplest case of optimization task with constraints in the form of inequalities, since it is required to minimize the simplest linear function $\mathrm{z}=\mathrm{f}$. Constraints in the form of inequalities can always be transformed into constraints in the form of equalities by adding positive reducing coefficients

$u_{i j}^{2}$ and $u_{i j}^{\prime 2}$ to each of them. Then, the task is reduced to minimization of function $\mathrm{z}=\mathrm{f}$ with existence of $2 n^{2}$ constraints of the form:

$$
\begin{gathered}
\stackrel{m o n}{q_{i j}}(f)+u_{i j}^{2}-(1+\varsigma) L q_{i j}=0 \\
D q_{i j}^{m o n}(f)+u_{i j}^{22}-(1+\eta) D q_{i j}=0 \\
i=\overline{1, n}, j=\overline{1, n} .
\end{gathered}
$$

Writing the Lagrange function with accounting for our constraints:

$$
F(f, \sigma, u)=f+\sum_{i, j=1}^{n} \sigma_{i j}^{\prime}\left[L q_{i j}^{m o n}(f)+u_{i j}^{\prime 2}-(1+\varsigma) L q_{i j}\right]+\sigma_{i j}^{\prime \prime}\left[D q_{i j}^{m o n}(f)+u_{i j}^{\prime 2}-(1+\eta) D q_{i j}\right]
$$

where $\sigma$ is the Lagrange multiplier, we obtain the following required conditions, which should be met in stationary point: 


$$
\begin{gathered}
\frac{\partial F}{\partial f}=\sum_{i, j=1}^{n} \sigma_{i j}^{\prime} \frac{\partial L q_{i j}^{m}(f)}{\partial f}+\sigma_{i j}^{\prime \prime} \frac{\partial D q_{i j}(f)}{\partial f}=0, \\
\frac{\partial F}{\partial \sigma_{i j}^{\prime}}=L q_{i j}^{m o n}(f)+u_{i j}^{\prime 2}-(1+\varsigma) L q_{i j}=0, \\
\frac{\partial F}{\partial \sigma_{i j}^{\prime \prime}}=D q_{i j}^{m o n}(f)+u_{i j}^{\prime 2}-(1+\eta) D q_{i j}=0 \\
\sigma_{i j}^{\prime}\left[(1+\varsigma) L q_{i j}-L q_{i j}^{m o n}(f)\right]=0 \\
\sigma_{i j}^{\prime \prime}\left[(1+\eta) D q_{i j}-D q_{i j}^{m o n}(f)\right]=0 \\
i=\overline{1, n}, j=\overline{1, n}
\end{gathered}
$$

If our constraints (or a part of them) exist in the form of strict inequality, then the Lagrange multipliers are zero (or a part of Lagrange multipliers corresponding to these constraints).

Optimization task can be expanded to asynchronous case, and the model of telecommunication network does not vary, since initially the monitoring packets generated by devices can be readily taken into account by additional flow $\lambda^{(3)}$. The optimization task should be reduced to detection of monitoring frequency $f_{\text {mon }}$ (for one controlling station) and densities of monitoring flows of individual devices, directly depending on data amount sent by this device to monitoring station.

In this case the optimization task will be reduced to minimization of certain function, which will depend on monitoring frequency $\mathrm{f}_{\mathrm{mon}}$ and densities of monitoring flows $\lambda_{i j}^{(2)}$ :

$$
g=f\left(f_{\text {mon }}, \lambda_{1,1}^{(2)}, \ldots, \lambda_{1, n}^{(2)}, \lambda_{2, n}^{(2)}, \ldots, \lambda_{n, n}^{(2)}\right) .
$$

The conditions implied in this case are completely analogous to one-dimensional optimization task considered above with addition of new variables:

$$
\begin{gathered}
\underset{q_{i j}^{m o n}}{L}\left(f_{m o n},, \lambda_{1,1}^{(2)}, \ldots, \lambda_{1, n}^{(2)}, \lambda_{2, n}^{(2)}, \ldots, \lambda_{n, n}^{(2)}\right) \leq(1+\varsigma) L q_{i j} \\
D q_{i j}^{\text {mon }}\left(f_{m o n}, \lambda_{1,1}^{(2)}, \ldots, \lambda_{1, n}^{(2)}, \lambda_{2, n}^{(2)}, \ldots, \lambda_{n, n}^{(2)}\right) \leq(1+\eta) D q_{i j} \\
i=\overline{1, n}, j=\overline{1, n} .
\end{gathered}
$$

Such task is referred to multidimensional optimization tasks with constraints in the form of inequalities. Solution of such task can be simplified by searching for function minimum with respect to not all variables $\lambda_{i j}^{(2)}$, as well as by using linear minimized function $\mathbf{f}$, thus obtaining linear optimization task. 


\section{SYSTEM ARCHITECTURE ON THE BASIS OF SNMP (MONITORING OF TERMINAL EQUIPMENT IN RAILWAY SECTOR)}

All units of railway system cannot operate without wide application of various types of communication, which are arranged via respective lines. Cable route is most widely used for railway sector, it is the most efficient for safety, high quality and reliability of communications. Management of all types of communication at current stage is impossible without various data systems.

On the basis of the existent drawbacks of distributed monitoring system, in particular errors generated upon measurements of electrical parameters of cable communication lines by module instrumentation, it becomes necessary to develop automated data system including monitoring software, hardware, diagnostic instrumentation, and developed moisture detector with hygrometer. The developed system makes it possible to accurately monitor condensation in cable structures, directly influencing on confidence of received data on electrical parameters of copper long distance and local cable communications.

The developed data system makes it possible to expand functional capabilities of ECMA module of distributed monitoring system, TRS Manager, to expand monitoring capabilities of module diagnostic instrumentation, thus accelerating data processing by operator. It would eliminate human errors upon classification of this or that emergency situation and finally improve performances of overall sector.

Management protocols (communication protocols) are referred to application layer protocols of sevenlayer model of open system interconnection. The main purpose of these protocols is transfer of controlling actions from manager to agent, as well as transfer of receipt/confirmation of results obtained by the controlling action. Therefore, the protocols support management information model TMN, though, properly, they can be considered as alternative management technologies of communication devices and networks.

SNMP determines network as a combination of network management stations and network elements (host computers, gateways and routers, terminal servers), which jointly provide administrative interconnections between network managing stations and network agents.

SNMP is the most popular management protocol of various commercial, campus, and research interconnected networks. The concept of management and administration for networks using TCP/IP is regulated by RFC 1157 «Simple Network Management Protocol (SNMP)» (Case, Fedor, Schoffstall and Davin, 1990).

At present there are three versions of SNMP (Kirch, 2017): SNMP Version 1 (SNMP v1), SNMP Version 2 (SNMP v2), SNMP Version 3 (SNMP v3). These versions have much in common, however, SNMP v2 has some advantages, for instance, additional protocol operation capabilities. SNMP v3 mainly provides protection to SNMP and improvements in remote configuration.

User program, known as network manager, provides virtual interconnection with program known as SNMP agent. SNMP agent is located on remote network device and provides information to manager about the state of given device. SNMP agents make information available for Network Management Systems (NMS) on the basis of SNMP. This model as a structure of data subsystem of monitoring management is illustrated in Fig. 6. 


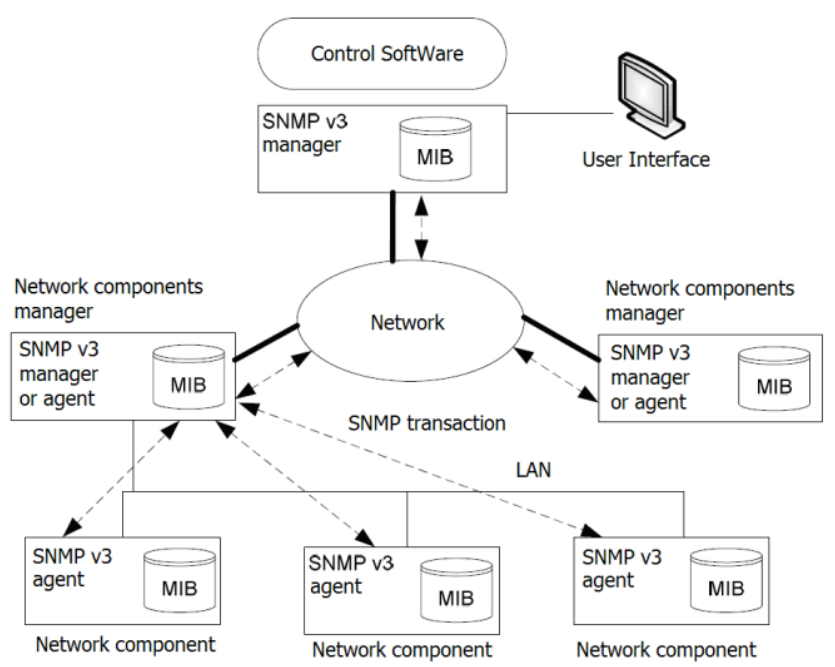

Figure 6. Schematic diagram of data subsystem of monitoring management using SNMP: MIB management information base.

Agents according to assignment from manager or automatically should trace the following operation performances of network equipment:

- amount and state of its virtual channels;

- number of certain failure messages;

- number of input and output bytes and packets of the given device;

- maximum output queue length (for routers and other devices);

- $\quad$ sent and received broad band messages;

- failed and restarted network interfaces.

\section{CONCLUSION}

The considered approach to mathematical simulation of data networking with packet routing makes it possible to analyze telecommunication networks with non-stationary flows. In addition, it is also possible to study flows with various components, in this case the interest was attracted to monitoring of data networking, hence, the traffic was subdivided into three components: $\lambda^{(0)}$ - common traffic (packet structure from various applications was of no concern), $\lambda^{(1)}-$ monitoring system traffic, and $\lambda^{(2)}$ asynchronous monitoring traffic generated by devices. The simulation was aimed at selection of optimum parameters of monitoring system, solutions of such task were proposed.

The obtained optimization task is sufficiently complicated, further purpose is searching for conditions which would allow to introduce some constraints in order to simplify its solution without noticeable loss of the model adequacy. This is especially important for asynchronous task of monitoring, it should be based on selection of the most acceptable type of objective function (linear or quadratic). In addition, it could be necessary to introduce additional optimization task for determination of optimum number of devices in telecommunication network, which generate asynchronous monitoring traffic. Then, it could be 
possible to determine sufficient conditions of minimum of our objective function. No less important is analysis of the model for adequacy using a priori experimental data.

The developed structure of data system management makes it possible to expand significantly functional capabilities of existing distributed monitoring system. First of all, the developed system would permit to improve confidence level of data received from measuring system about electrical parameters of route cables, thus decreasing human errors upon classification of emergency situations occurring in communication networks. Flexible reporting system implemented in the monitoring and administration system makes it possible to determine and solve bottleneck problems and to prevent probable failures.

Tracing of personnel actions upon their operation with network using the tools of distributed system makes it possible to reveal not only negative events but best practices, to arrange reasonable measures aiming at improvement of operation quality.

Inventory management in system of not only equipment and network infrastructures but also of generated upon network operation virtual objects (such as tracks and channels with fixed bandwidth) makes it possible to obtain distinct pattern of influence of processes related with network operation on quality of rendered services.

\section{REFERENCES}

Ahn, S., Chung, J.W. (2005). A study upon SNMP MIB II algorithm: For the calculation of the line utilization. The Journal of Supercomputing, 33, 79.

Bandi, B. (1988). Optimization methods. Introductory course, Radio communications. Moscow.

Case, J., Fedor, M., Schoffstall, M., Davin, J. (1990). A Simple Network Management Protocol (SNMP). Retrieved from: https://www.ietf.org/rfc/rfc1157.txt.

Clarke, A.B. (1957). A Waiting Time Process of Markov Type, Ann. Math. Statist., 27, 452-459.

Douglas, P. (1983). Kingston III, A Tour Through the Multi-Device Queueing System, revised for MDQS 2.0, Ballistic Research Laboratory, Army Armament Research and Development Command (AARADCOM).

Fletcher, R. (2000). Practical methods of Optimization, Wiley.

Gabor, L., Istvan, D., Laszlo, M. (2013). Towards the efficient simulation of telecommunication systems in heterogeneous distributed execution environments, in Proc. of Telecommunications and Signal Processing (TSP), 36th International Conference on.

Jackson, J.R. (1957). Networks of Waiting Lines, Operations Research, 5(4), 518-521.

Khan, M.A., Imtiaz, S.Y., Shakir, M. (2015). Automatic Monitoring \& Detection System (AMDS) for Grey Traffic, in Proc. of the World Congress on Engineering and Computer Science 2015, Vol II, WCECS 2015, October 21-23, San Francisco, USA, pp. 696-700.

Kirch, A. (2017). “SNMP”, Linux Journal, Jan 24, 2017 http://www.linuxjournal.com/content/snmp

Kitchin, J. (2006). Approximate Markov Modeling of High-Reliability Telecommunications Systems, IEEE Journal on Selected Areas in Communications, 4(7), 1133-1137. 
Kuai K., Tsai C., 2016. Discrete-Time Markov Chain Model for Transport of Mixed-Size Sediment Particles under Unsteady FlowConditions. Journal of Hydrologic Engineering, 21(11).

Merck, J.W., Hall, K. (1971). A Markovian flow model: the analysis of movement in large-scale (military) personnel systems. Rand Corp., R-514-PR. Retrieved from: https://www.rand.org/content/dam/rand/pubs/reports/2007/R514.pdf

Morley, C.D., Thornes, J.B. (2010). A Markov Decision Model for Network Flows. Geographical Analysis, 4(2).

Norris, J.R. (1997). Continuous-time Markov chains I, Markov Chains, pp. 60.

Pandey, S., Choi, M.-J., Won, Y.J., Hong, J.W.K. (2011). SNMP-based enterprise IP network topology discovery, Int. J. Network Mgmt., 21(3), 169-184.

Romansky, R. (2013). An Application of Procedure for Analytical Evaluation of Computer Structures, International Journal on Information Technologies and Security, 5/2013, 55.

Spajic, V., Kozic, N., Pokrajac, I., Okiljevic, P. (2012). Radio monitoring of telecommunication systems with TDMA multiple access technique, in Proc. of Telecommunications Forum (TELFOR), $20^{\text {th }}$.

Subramanian, R., Raman, P., George, A.D., Radlinski, M.A. (2006). Gossip Enabled monitoring services for scalable heterogenous distributed systems. Cluster computing, 9(1), 101-120.

Tyagi, S., Tanwar, S., Gupta, S.K., Kumar, N., Rodrigues, J.J.P.C. (2015). A lifetime extended multilevels heterogeneous routing protocol for wireless sensor networks, Telecommunication Systems, 59, 4362. 